\title{
Capacitated Vehicle Routing Problem for Thailand's Steel Industry via Saving Algorithms
}

\author{
Siwaporn Kunnapapdeelert, Chananya Thawnern \\ International College, Burapha University, Chonburi, 20131, Thailand. \\ siwapornk@go.buu.ac.th
}

\begin{abstract}
Goal of supply chain management is to effectively manage the physical flow, information flow and financial flow from upstream to downstream for satisfying the customer need. Transportation and distribution of inventory becomes an important activity in supply chain in real-world competitive situation. Vehicle routing problem (VRP) is NP-hard problem, which is complex and difficult to solve. Some companies design the route of vehicle by using an experience of the planner however; the designing of the planner might not provide good solution. Heuristics approach is found to be an effective approach to solve VRP. The aim of this research is to apply Clarke and Wright's saving algorithm for designing the vehicle route for steel industry distribution. Capacitated vehicle routing problem (CVRP) from the real-world problem is used for testing the effectiveness of the proposed algorithm in this study. The flatbed trailer with the maximum capacity of 25 tons is considered as the vehicle used in this problem. The tested problem composes of only one depot and 15 randomly distributed customers' location with different demand. The vehicle routes design from human's experience is compared to the vehicle routes designed based on Clarke and Wright's saving algorithm. The experimental results reveal that the proposed algorithm provides the superior results to the route planner's experience. The results obtained from the proposed algorithm provide 8.08 percent better than the results from the traditional approach. The reason that the proposed approach provides the better results is mainly from the distance saving for each pair of customers process couple with the saving list construction in Clarke and Wright's saving algorithm.
\end{abstract}

Keywords: Capacitated vehicle routing, distribution; saving algorithm, heuristics, optimization, logistics. 


\section{Introduction}

Thailand is a developing country that needs to develop basic infrastructure across the country. Thai government has endorsed several projects for promoting the major infrastructure projects in 2021. Further, the government has created a project called Eastern Economic Corridor (EEC) to develop eastern part of the country to be the center of technological manufacturing and services which would push domestic steel consumption to grow. An increase in demand for steel means that the need for steel delivery from upstream to downstream is increasing. Various companies pay a lot of transportation costs for transferring their goods from one place to another place. A good design of vehicle routes can reduce the transportation expenses that come from transportation distance and transportation time. It also enhances service ability while increases customer satisfaction level at the same time. Consequently, numerous logistics practitioners and researchers have been tried to find the way to determine the optimal transportation routes to deliver the products to the customers.

In the steel industry, finished steel products can be categorized into two main products as long products and flat products. The long products such as steel bar and hot-rolled (HR) section are used for construction while wired rod and seamless pipe are generally used for both construction and industries. In the case of flat products, hot-rolled coil (HRC) is used for industries while cold-rolled coil (CRC), metal coating, and welded pipe are used in both industries and the construction sector. The shape and size of such products are not normal and cannot fit into the container, therefore the flatbed trailers are the vehicle used for delivering such products. In this research, the distribution of steel products of midstream and downstream in the supply chain is studied.

The design of trailer routes is implemented based on the heuristics approach namely Clarke and Wright's savings algorithm. It is applied for improving efficiency while reducing transportation cost and time. Previously, the transportation route planning was designed by the senior route planner that has experience in the route by searching for the directions and distances from google map and then assigning the route for each driver. This traditional route planning is time consuming and costly which needs to be improved. Therefore, the design of the vehicle routing for delivery steel in Thailand by using heuristic approach, Clarke and Wright's savings algorithm, for enhancing the transportation efficiency is the purpose of this work.

\section{Literature Review}

Heuristics method is an approach that employs for solving practical problems which does not guarantee the optimal solution. However, it reduces the mental effort needed of the decision maker. It helps to simplify the complex problems and difficult questions. This method is a fast and accurate approach to reach the conclusion. Heuristics was firstly introduced by psychologists Simon (1947) for human choice and administrative decision-making to manage with organizational problems. 
Heuristics is one of an efficient approach in problem-solving, planning, and decisionmaking in complex problems of real-world practice (Leritz, 2005). Several researchers have applied metaheuristics approach for solving problems in various fields such as social science (Heath et al., 2013), finance (Gilli, 2008), healthcare (Whelehan et al., 2020), engineering (Daly, 2012), computer science and operations research (Hromkovič, 2013), etc. Heuristics is widely used to determine the reasonably accurate solutions of the hard problems including travelling salesman problem, scheduling, network design problem and facility location.

Vehicle Routing Problem (VRP) is an extension of Travelling Salesman Problem (TSP). It is one of the most challenging combinatorial optimization problems. Dantzig and Ramsey first proposed VRP in 1959. The objective of VRP is to evaluate the optimal route for multiple vehicles by minimizing total distance under operational or business-specific constraints. Those constraints are cost control, vehicle limitations (size and weight), route distance, and time window. This research emphasizes the capacitated vehicle routing problem (CVRP) which is a classical version of VRP. The pick up and delivery activities must be completed in one trip. All vehicles must start and end its tour at the same depot. The vehicle must service to the known demand customers under the following limitations:

- every route starts and ends at the same depot,

- each customer must be visited once by only one vehicle,

- total demand in each route must not exceed the vehicle capacity,

- the total time of each route composes of transportation time, service time, which is not exceed the predefined limitation,

- the total transportation cost is minimized.

Saving algorithm was introduced by Clarke and Wright (1964) for solving VRP. It has been successfully applied for solving VRP in real world practice cases. Clarke and Wright algorithm is then explained by Laported (1992) as follows.

Step 1: Compute the savings $s_{i j}=c_{i 1}+c_{1 j}-c_{i j}$ for $i, j=2, \ldots, n$ and $i \neq j$. Generate $n-1$ vehicle routes $(1, i, 1)(i=2, \ldots, n)$.

Step 2: Order the savings in a non-increasing fashion.

Step 3: Consider two vehicle route that contain arcs $(i, 1)$ and $(1, j)$, respectively. If $s_{i j}>0$, tentatively merge these routes by introducing arc $(i, j)$ and by removing $\operatorname{arcs}(i, 1)$ and $(1, j)$. Implement the merge it the resulting route is feasible.

Step 4: Repeat step 3 until no further improvement is possible. Stop.

However, Christofides et al. (1979) extended the explanation of Clarke and Wright in steps 3 and 4 by classifying the method to arrange the routes into two different approaches as parallel and sequential versions as explained below.

- Parallel Version:

(i) If the given link is a feasible route based on the VRP constraints, then add this link to the solution, if not reject the link. 
(ii) Try the next link in the list then repeat (i) until no more link can be selected.

- Sequential Version:

(i) Determine the first link in the list that can extend one of two ends of the route that is currently generated.

(ii) If the route cannot further extend, or no route exists, select the first feasible link in the list to create the new route.

(iii) Repeat steps (i) and (ii) until no more links can be selected.

Several researchers have been successfully applied these two different versions of saving algorithm for solving VRP. Cordeau et al., (2002) found that parallel versions provided the superior results to those of sequential versions. However, Rand (2009) argued that parallel version does not always provide the better results than the sequential version. It is because the saving algorithm is the heuristics approach which does not guarantee the optimal solutions. However, the parallel version of Clarke and Wright's saving algorithm usually provides the better results than the sequential version (Pichpibul \& Kawtummachai, 2013). Consequently, the parallel version of Clarke and Wright's savings algorithm is selected for solving CVRP to design the vehicle routing for delivery steel in this work. Clarke and Wright's savings algorithm can be summarized to be four main steps 1) establish distance matrix, 2) determine the saving matrix, 3) assign customers to each route, and 4) sequence customers in the route.

\section{Methodology}

The purpose of this research is to apply the heuristics approach for enhancing efficiency of vehicle route planning to deliver the steel products in Thailand. The vehicle route from traditional method which obtained from an experience of senior transport planer of the company was found to be time consuming and costly. Subsequently, the saving algorithm is applied to improve the vehicle route planning ability of the company. The routes obtained from the traditional approach are compared to the planned routes from Clarke and Wright's saving algorithm based on the restriction that maximum capacity of each trailer is not exceeded 25 tons. The information of distance between location of origin and destination including demand of each customer are described below.

The trailers are started and ended at the same terminal $(\boldsymbol{T})$, goods are distributed to 15 different customers $(\boldsymbol{A}-\boldsymbol{O})$. The demand of each customer and distance between each location are explained in Tables 1 and 2, respectively. 
Table 1. Demand of each customer in one day (tons).

\begin{tabular}{|c|c|c|c|}
\hline Customer & Demand (tons) & Customer & Demand (tons) \\
\hline A & 6 & I & 10 \\
\hline B & 6 & J & 5 \\
\hline C & 11 & K & 8 \\
\hline D & 7 & L & 3 \\
\hline E & 6 & M & 8 \\
\hline F & 10 & N & 7 \\
\hline G & 9 & O & 10 \\
\hline H & 10 & & \\
\hline
\end{tabular}

Table 2. Distance between customers (kilometers)

\begin{tabular}{|c|c|c|c|c|c|c|c|c|c|c|c|c|c|c|c|c|}
\hline $\begin{array}{c}\text { From } \\
\text { /To }\end{array}$ & $\mathbf{T}$ & $\mathbf{A}$ & $\mathbf{B}$ & $\mathbf{C}$ & $\mathbf{D}$ & $\mathbf{E}$ & $\mathbf{F}$ & $\mathbf{G}$ & $\mathbf{H}$ & $\mathbf{I}$ & $\mathbf{J}$ & $\mathbf{K}$ & $\mathbf{L}$ & $\mathbf{M}$ & $\mathbf{N}$ & $\mathbf{O}$ \\
\hline $\mathbf{T}$ & 0 & - & - & - & - & - & - & - & - & - & - & - & - & - & - & - \\
\hline $\mathbf{A}$ & 55 & 0 & - & - & - & - & - & - & - & - & - & - & - & - & - & - \\
\hline $\mathbf{B}$ & 15.2 & 40.1 & 0 & - & - & - & - & - & - & - & - & - & - & - & - & - \\
\hline $\mathbf{C}$ & 23.2 & 41.9 & 21.2 & 0 & - & - & - & - & - & - & - & - & - & - & - & - \\
\hline $\mathbf{D}$ & 123 & 84.7 & 98.7 & 114 & 0 & - & - & - & - & - & - & - & - & - & - & - \\
\hline $\mathbf{E}$ & 21.7 & 40.6 & 5.3 & 28.9 & 99.8 & 0 & - & - & - & - & - & - & - & - & - & - \\
\hline $\mathbf{F}$ & 13.5 & 44.5 & 5.1 & 14.9 & 103 & 7.2 & 0 & - & - & - & - & - & - & - & - & - \\
\hline $\mathbf{G}$ & 17 & 47.8 & 9.3 & 10.5 & 106 & 16.3 & 6 & 0 & - & - & - & - & - & - & - & - \\
\hline $\mathbf{H}$ & 57.7 & 9.6 & 34.1 & 38.1 & 86.3 & 34.6 & 38.4 & 45.4 & 0 & - & - & - & - & - & - & - \\
\hline $\mathbf{I}$ & 23.3 & 42 & 27.9 & 0.09 & 114 & 29 & 15 & 10.6 & 42 & 0 & - & - & - & - & - & - \\
\hline $\mathbf{J}$ & 23.3 & 44.6 & 19.4 & 5.6 & 111 & 20.7 & 16.5 & 12.2 & 45.4 & 5.7 & 0 & - & - & - & - & - \\
\hline $\mathbf{K}$ & 53.3 & 27.5 & 36.2 & 46.4 & 68 & 36.6 & 42.8 & 47.9 & 27.3 & 46.5 & 49.1 & 0 & - & - & - & - \\
\hline $\mathbf{L}$ & 23.4 & 42.1 & 19.5 & 0.22 & 114 & 29.1 & 15.1 & 10.7 & 45.4 & 0.13 & 5.2 & 54.2 & 0 & - & - & - \\
\hline $\mathbf{M}$ & 65.5 & 15 & 51.1 & 57.4 & 82.6 & 52.2 & 55.4 & 54.2 & 20.4 & 57.5 & 54.1 & 31.8 & 57.7 & 0 & - & - \\
\hline $\mathbf{N}$ & 25.8 & 41.4 & 27.3 & 2.2 & 113 & 28.4 & 17.5 & 13.2 & 37.7 & 2.3 & 3.3 & 54.2 & 2.4 & 55.8 & 0 & - \\
\hline $\mathbf{O}$ & 191 & 138 & 171 & 182 & 140 & 173 & 176 & 183 & 145 & 182 & 179 & 133 & 182 & 136 & 180 & 0 \\
\hline
\end{tabular}

The flow chart of Clarke and Wright's saving algorithm that is used in this work is described in Figure 1.

The percentage improvement is used for analyzing the performance of the proposed algorithm which can be calculated as follows:

$$
\text { Percentage improvement }=\left(\frac{\text { Traditional-Saving }}{\text { Traditional }}\right) \times 100 .
$$

\section{Results and Discussions}

Two different approaches for designing vehicle routes are compared. The traditional distance is determined based on an experience of the senior transport planner via google map. The planned route from the traditional approach is described in Table 3. 
Table 3. Solution from the traditional approach based on the senior transport planner's experience.

\begin{tabular}{|c|c|c|c|}
\hline & Trip & Length of trip (km.) & Load on truck (tons) \\
\hline Route 1 & T-M-D-O-T & 479.1 & 25 \\
\hline Route 2 & T-A-K-C-T & 152.1 & 25 \\
\hline Route 3 & T-H-N-J-T & 122 & 22 \\
\hline Route 4 & T-I-L-G-T & 51.13 & 22 \\
\hline Route 5 & T-B-F-E-T & 49.2 & 22 \\
\hline & & 853.53 & 116 \\
\hline
\end{tabular}

The CVRP is later solved by Clarke and Wright's saving algorithm. Length of trip for each and load in each truck is determined as the first step. It can be calculated from the saving pairs based on the $\mathrm{CW}$ (Saving algorithm) which can be calculated as distance $\left(T-C_{i}\right)+$ distance $\left(T-C_{j}\right)$ - distance $\left(C_{i}-C_{j}\right)$. According to the saving equation, the saving distance can be determined as follows. 


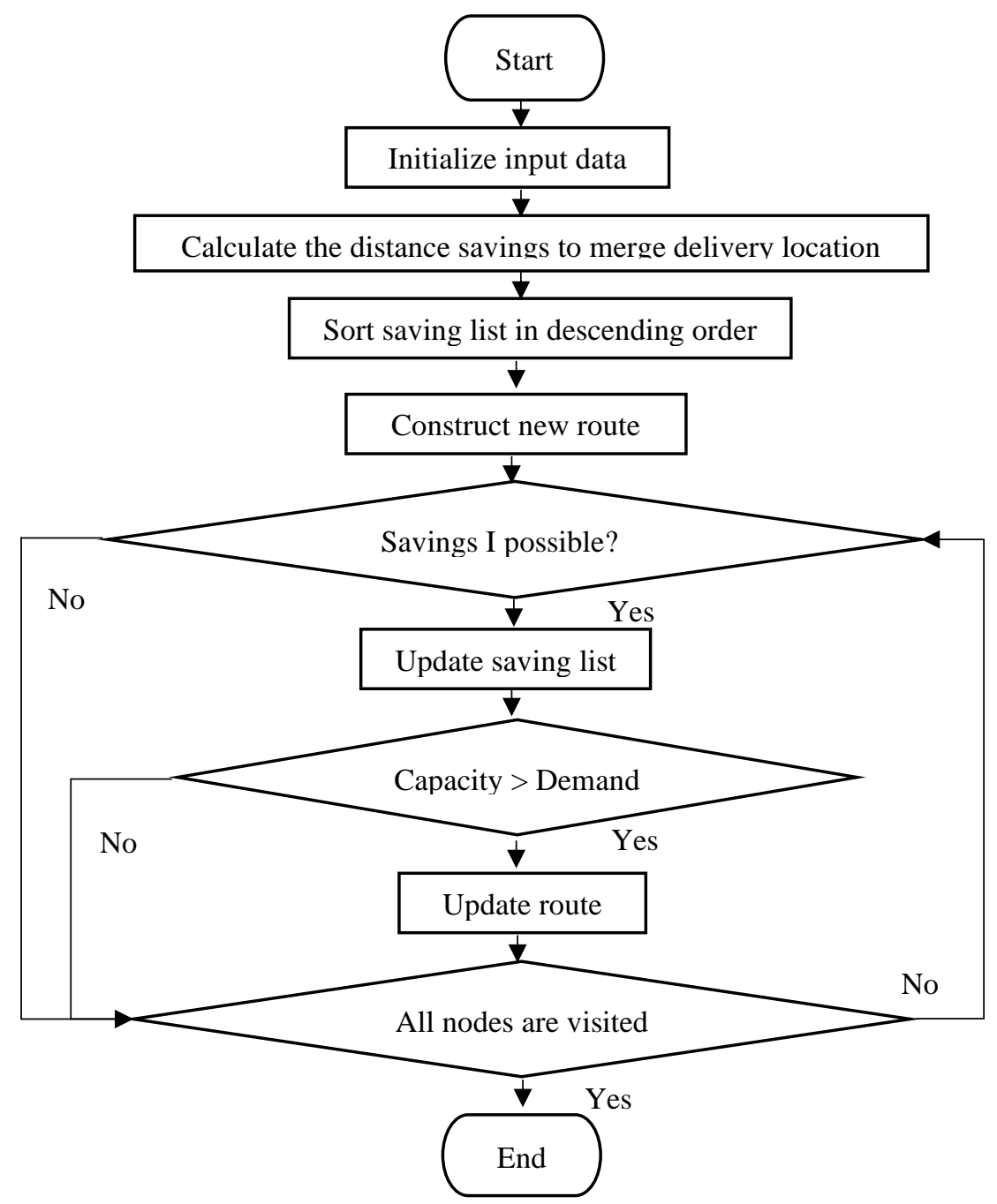

Fig.1. Flow chart of Clarke and Wright's saving algorithm.

Given that distance between $T$ and $A$ is $\overline{T A}(=55)$, distance between $T$ and $B$ is $\overline{T B}$ (15.2) and distance between $A$ and $B$ is $\overline{A B}(=40.1)$ as presented in Table 4.

$$
\overline{2 T A}+\overline{2 T C}-(\overline{T A}+\overline{A C}+\overline{T C})=\overline{T A}+\overline{T C}-\overline{A C}=55+23.2-41.9=36.3 .
$$

Saving distance for every pair of customers are calculated based on the same concept as summarized in Table 4.

If customers $A$ and $B$ are in the same trip that served from $T$ then the following distance is saved:

$$
\overline{2 T A}+\overline{2 T B}-(\overline{T A}+\overline{A B}+\overline{T B})=\overline{T A}+\overline{T B}-\overline{A B}=55+15.2-40.1=30.1 .
$$

If customers $B$ and $C$ are in the same trip that served from $T$ then the following distance is saved: 


$$
\overline{2 T B}+\overline{2 T C}-(\overline{T B}+\overline{B C}+\overline{T C})=\overline{T B}+\overline{T C}-\overline{B C}=15.2+23.2-21.2=17.2 \text {. }
$$

If customers $A$ and $C$ are in the same trip that served from $T$ then the following distance is saved:

Table 4. Distance saving for each pair of customers (kilometers).

\begin{tabular}{|c|c|c|c|c|c|c|c|c|c|c|c|c|c|c|c|}
\hline $\begin{array}{c}\text { From } \\
\text { /To }\end{array}$ & $\mathrm{A}$ & $\mathrm{B}$ & $\mathrm{C}$ & $\mathrm{D}$ & $\mathrm{E}$ & $\mathrm{F}$ & $\mathrm{G}$ & $\mathrm{H}$ & $\mathrm{I}$ & $\mathrm{J}$ & $\mathrm{K}$ & $\mathrm{L}$ & $\mathrm{M}$ & $\mathrm{N}$ & $\mathrm{O}$ \\
\hline $\mathrm{A}$ & 0 & - & - & - & - & - & - & - & - & - & - & - & - & - & - \\
\hline $\mathrm{B}$ & 30.1 & 0 & - & - & - & - & - & - & - & - & - & - & - & - & - \\
\hline $\mathrm{C}$ & 36.3 & 17.2 & 0 & - & - & - & - & - & - & - & - & - & - & - & - \\
\hline $\mathrm{D}$ & 93.3 & 39.5 & 32.2 & 0 & - & - & - & - & - & - & - & - & - & - & - \\
\hline E & 36.1 & 31.6 & 16 & 44.9 & 0 & - & - & - & - & - & - & - & - & - & - \\
\hline F & 24 & 23.6 & 21.8 & 33.5 & 28 & 0 & - & - & - & - & - & - & - & - & - \\
\hline G & 24.2 & 62.7 & 29.7 & 34 & 22.4 & 24.5 & 0 & - & - & - & - & - & - & - & - \\
\hline H & 103.1 & 38.8 & 42.8 & 94.4 & 44.8 & 32.8 & 29.3 & 0 & - & - & - & - & - & - & - \\
\hline I & 36.3 & 10.6 & 46.41 & 32.3 & 16 & 21.8 & 29.7 & 39 & 0 & - & - & - & - & - & - \\
\hline J & 33.7 & 19.1 & 40.9 & 35.3 & 24.3 & 20.3 & 28.1 & 35.6 & 40.9 & 0 & - & - & - & - & - \\
\hline K & 80.8 & 32.3 & 30.1 & 108.3 & 38.4 & 24 & 22.4 & 83.7 & 30.1 & 27.5 & 0 & - & - & - & - \\
\hline L & 36.3 & 19.1 & 46.38 & 32.4 & 16 & 21.8 & 29.7 & 35.7 & 46.57 & 41.5 & 22.5 & 0 & - & - & - \\
\hline M & 105.5 & 29.6 & 31.3 & 105.9 & 35 & 23.6 & 28.3 & 102.8 & 31.3 & 34.7 & 87 & 31.2 & 0 & - & - \\
\hline N & 39.4 & 13.7 & 46.8 & 35.8 & 19.1 & 21.8 & 29.6 & 45.8 & 46.8 & 45.8 & 24.9 & 46.8 & 35.5 & 0 & - \\
\hline O & 108 & 35.2 & 32.2 & 174 & 39.7 & 28.5 & 25 & 103.7 & 32.3 & 35.3 & 111.3 & 32.4 & 120.5 & 36.8 & 0 \\
\hline
\end{tabular}

The first route (Route 1: T-D-O-M-T), the saving distance is $123+140+136+$ $65.5=464.5$ kilometers. Load of on truck for route 1 can be calculated as $7+10+8$ $=25$ tons.

The second route (Route 2: T-A-H-K-T), the saving distance is $55+9.6+27.3+$ $53.3=145.2$ kilometers. Load of on truck for route 2 can be calculated as $6+10+8$ $=24$ tons.

The third route (Route 3: T-F-E-J-T), the saving distance is $13.5+7.2+20.7+$ $23.3=64.7$ kilometers. Load of on truck for route 1 can be calculated as $10+6+5$ $=21$ tons.

The fourth route (Route 4: T-B-G-N-L-T), the saving distance is $15.2+9.3+13.2$ $+2.4+23.4=63.5$ kilometers. Load of on truck for route 2 can be calculated as $6+$ $9+7+3=25$ tons.

The last route (Route 5: T-I-C-T), the saving distance is $23.3+0.09+23.2=46.59$ kilometers. Load of on truck for route 2 can be calculated as $10+11=21$ tons.

The summarize of the solution from CW (Saving algorithm) is presented in Table 5 . 
Table 5. Solution from CW (Saving algorithm).

\begin{tabular}{|l|l|c|c|}
\hline & \multicolumn{1}{|c|}{ Trip } & Length of trip $(\mathrm{km})$. & Load on truck (tons) \\
\hline Route 1 & T-D-O-M-T & 464.5 & 25 \\
\hline Route 2 & T-A-H-K-T & 145.2 & 24 \\
\hline Route 3 & T-F-E-J-T & 64.7 & 21 \\
\hline Route 4 & T-B-G-N-L-T & 63.5 & 25 \\
\hline Route 5 & T-I-C-T & 46.59 & 21 \\
\hline & & 784.49 & 116 \\
\hline
\end{tabular}

The route planning based on senior trailer planner's experience compose of 5 different routes with total distance of 853.53 kilometers. The results obtained by Clarke and Wright's saving algorithm consist of 5 different routes which provides total distance of 784.49 kilometers. Further, it was found that total distance from the Clarke and Wright's saving algorithm is improved from those of traditional approach 69.04 kilometers or around 8.09 percent improvement. The reason that total travel distances from the proposed algorithm are shorter than those of the traditional approach is mainly from the determination of distance saving for each pair of customers and saving list construction steps. These processes enhance quality of the solution by combining two routes into one so-called pooling. This confirms that Clarke and Wright's saving algorithm provider superior results to the traditional approach. Transportation time and cost are directly proportional to the travel distance. As the total travelled distance of the trailers are reduced, the transportation time and cost are also declined.

\section{Conclusions and Recommendations}

The purpose of this research is to design the optimal route to distribute finished steel products with the restriction of vehicle capacity and number of flatbed trailers by using heuristics approach. The experiment was conducted based on the real-world data from Thailand's steel industry. This data is classified as CVRP which is NP hard problem that can be exactly solved by only for small problems. NP-hard problem is known to be effectively solved by heuristics-based approach. Previously, the vehicle route design was conducted by using an experience of the route planner couple with the use of google map for finding the minimum route of the vehicles. However, the design of CVRP is time consuming and does not optimize the transportation cost. Consequently, Clarke and Wright's saving algorithm is proposed to design the route for distributing the products in this work. It is because the proposed approach is easy to understand and implement comparing to the meta-heuristic approaches. The experimental results shown that Clarke and Wright's saving algorithm provides the better solutions than those of the traditional approach up to 8.09 percent improvement. This confirms that Clarke and Wright's saving algorithm is successfully applied for designing the vehicle route in the real-world industry. This approach is also able to reduce transportation cost and time. It would be more beneficial to apply the proposed 
method for solving the larger and more complicated problems.

The limitation of this work is the number of real worlds tested case is still limited. The proposed method is recommended to be an approach for solving CVRP when size of the problems is larger. It would be more effective when other optimization search techniques are combined to the Clarke and Wright's saving algorithm for solving CVRP.

\section{References}

Simon, H. A. (1947). Administrative behavior. New York: Macmillan.

Leritz, L. E. (2005). Michael D. Mumford. Encyclopedia of Social Measurement, 2, 203.

Gora, P., Bankiewicz, D., Karnas, K., Kaźmierczak, W., Kutwin, M., Perkowski, P., Plotka, S., Szczurek, A., \& Zięba, D. (2020). On a road to optimal fleet routing algorithms: a gentle introduction to the state-of-the-art. In Smart Delivery Systems (37-92). Elsevier.

Heath, L., Tindale, R. S., Edwards, J., Posavac, E. J., Bryant, F. B., Henderson-King, E., \& Myers, J. (Eds.). (2013). Applications of heuristics and biases to social issues. Springer Science \& Business Media, 3.

Gilli, M., Maringer, D., \& Winker, P. (2008). Applications of heuristics in finance. In Handbook on information technology in finance (pp. 635-653). Springer, Berlin, Heidelberg.

Whelehan, D. F., Conlon, K. C., \& Ridgway, P. F. (2020). Medicine and heuristics: cognitive biases and medical decision-making. Irish Journal of Medical Science (1971-), 189, 1477-1484.

Daly, S. R., Yilmaz, S., Christian, J. L., Seifert, C. M., \& Gonzalez, R. (2012). Design heuristics in engineering concept generation. Journal of Engineering Education, 101(4), 601-629.

Hromkovič, J. (2013). Algorithmics for hard problems: introduction to combinatorial optimization, randomization, approximation, and heuristics. Springer Science \& Business Media.

Dantzig, G. B., \& Ramser, J. H. (1959). The truck dispatching problem. Management Science, 6(1), 80-91. 
Clarke, G., \& Wright, J. W. (1964). Scheduling of vehicles from a central depot to a number of delivery points. Operations Research, 12(4), 568-581.

Laporte, G. (1992). The vehicle routing problem: An overview of exact and approximate algorithms. European Journal of Operational Research, 59(3), 345-358.

Christofides, N., \& Mingozzi, A. T. (1978). P. 1979. The vehicle routing problem. Combinatorial optimization. Chichester: Wiley, 315-338.

Cordeau, J. F., Gendreau, M., Laporte, G., Potvin, J. Y., \& Semet, F. (2002). A guide to vehicle routing heuristics. Journal of the Operational Research society, 53(5), 512522 .

Pichpibul, T., \& Kawtummachai, R. (2013). A heuristic approach based on clarkewright algorithm for open vehicle routing problem. The scientific world journal, 2013, 874349 .

Rand, G. K. (2009). The life and times of the Savings Method for Vehicle Routing Problems. ORiON, 25(2). 